Bundesgesundheitsbl 2015 $58: 341-344$

DOI 10.1007/s00103-015-2138-1

Online publiziert: 7. März 2015

(c) Springer-Verlag Berlin Heidelberg 2015

\author{
Martin Härter ${ }^{1}$ Uwe Koch-Gromus ${ }^{2}$ \\ ${ }^{1}$ Institut und Poliklinik für Medizinische Psychologie, Zentrum für Psychosoziale Medizin, \\ Universitätsklinikum Hamburg-Eppendorf, Hamburg, Deutschland \\ 2 Medizinische Fakultät, Campus Lehre, N 55, Universitätsklinikum Hamburg-Eppendorf, \\ Hamburg, Deutschland
}

\title{
Innovative Versorgungsformen und Modelle der vernetzten Versorgung
}

Eine effektive(re) Koordinierung der Versorgungs- und Betreuungsprozesse über die verschiedenen Leistungssektoren hinweg und eine stärkere Patientenorientierung sind in Deutschland Themen, welche die Akteure im Gesundheitswesen seit Jahrzehnten prioritär auf ihrer Agenda haben. Die nach wie vor bestehenden zahlreichen Schnittstellen und daraus resultierenden Versorgungsbrüche an den Sektorengrenzen, ein in den letzten Jahren zunehmender Wettbewerbsdruck unter den Leistungserbringern und regionale Variationen in der Gesundheitsversorgung können eine optimale Versorgung, insbesondere älterer, chronisch, multimorbid oder psychisch erkrankter Menschen behindern. Diese zeichnet sich in Deutschland vor allem durch eine hohe Zahl an jährlichen Arztkontakten und parallel bestehende Über-, Unter- und Fehlversorgung aus. Am deutlichsten treten diese Probleme beim Übergang vom stationären in den ambulanten Sektor auf. Aber auch innerhalb der Sektoren behindern Versorgungsbrüche sowie unzureichende Informationsflüsse eine patientenorientierte Versorgung. Sektoral vergebene Budgets, bestehende Fehlanreize im Gesundheitswesen, mangelnde Evidenzbasierung zahlreicher Prozesse sowie die nicht ausreichende Transparenz der Erbringung von Gesundheitsdienstleistungen etc. verschärfen diese Probleme.

1997 verabschiedete die Bundesregierung das 2. Neuordnungsgesetz der Gesetzlichen Krankenversicherung (GKV), um die medizinische Versorgung auf Grundlage von sog. Modellvorhaben ( $\$ 63$ SGB V) und spezifischen Strukturverträgen
( $\$ 73 a$ SGB V) weiterzuentwickeln. Eine stärkere Vernetzung von niedergelassenen Ärzten und stationären Einrichtungen wurde erstmals förderwürdig. Weitere Handlungsmöglichkeiten in der ambulanten Versorgung, z. B. durch den neu eingeführten \$140a-d SGB V für Integrierte Verträge (IV) mit Leistungserbringern, die eine verschiedene Leistungssektoren übergreifende Versorgung sicherstellen sollten, folgten. Am besten gelang dies in der Folge zunächst über zahlreiche pragmatische Verträge, die wirtschaftliche Vorteile für Krankenkassen und Leistungserbringer erbrachten (Beispiel: Gelenkersatz oder ambulantes Operieren).

Anfang 2002 trat das Gesundheitsstrukturgesetz (GSG) in Kraft, welches die Rechtsgrundlage für strukturierte Behandlungsprogramme, die Disease-Management-Programme (DMP), festschrieb. Ziel war eine möglichst flächendeckende Versorgung von chronisch kranken Versicherten der Gesetzlichen Krankenversicherung (GKV), die von evidenzbasierten Leitlinien begleitet wird. Auch hier war sowohl ein strukturierter und sektorenübergreifender Behandlungsablauf als auch eine verbesserte Kooperation mit dem Patienten vorgesehen. $\mathrm{Zu}$ nächst starteten DMP zu den Indikationen Diabetes mellitus Typ 2 und Brustkrebs, bis 2006 folgten weitere DMP zu Diabetes mellitus Typ 1, koronare Herzerkrankung $(\mathrm{KH})$, chronisch obstruktive Lungenerkrankung (COPD) und Asthma bronchiale. Während z. B. in den USA die Wirksamkeit im Vorfeld der DMP-Einführung anhand von hochwertigen, prospektiven und kontrollierten Studien un- tersucht wurde, wurde dies in Deutschland nicht umgesetzt.

International werden IV-Modelle oder DM-Programme oft entweder unter dem Begriff DMP oder auch Collaborative Care umgesetzt und beforscht. Bei der sog. gestuften Versorgung (Stepped Care) beginnt die Behandlung mit derjenigen Interventionsform, die leitliniengemäß adäquat ist und gleichzeitig die geringste Behandlungsintensität aufweist; bei Nichtansprechen wird die Behandlung mit der nächst höheren Intensitätsstufe fortgesetzt. Der Fokus liegt ebenfalls auf der verbesserten Vernetzung zwischen den an der Versorgung von chronisch oder psychisch kranken Patienten beteiligten Behandlern (z. B. Hausarzt, Psychiater, Psychotherapeut, Krankenpflege), um so Patienten eine leitlinienbasierte und bzgl. der Aufgaben verteilte und vernetzte Behandlung anzubieten.

Eine erfolgreiche Periode für die Integrierte Versorgung folgte ab 2004, als der neu formulierte $\$ 140 \mathrm{a}$-d SGB V vom Grundsatz der Beitragssatzstabilität befreit wurde, sogar $1 \%$ der Gesamtvergütung im GKV-System als Anschubfinanzierung zur Verfügung gestellt wurde. Mit dem GKV-Wettbewerbsstärkungsgesetz von 2007 wurden durch die Einbindung der Pflegeversicherung, der pharmazeutischen Industrie und der Medizinproduktehersteller weitere IV-Vertragspartner ermöglicht. Darüber hinaus wurde das SGB V um die Regelung einer „Hausarzt-zentrierten Versorgung" ( $\mathrm{HzV})$ in $\$ 73$ b ergänzt. Perspektivisch sollte diese $\mathrm{HzV}$ allerdings nicht alleine stehen, sondern, zusammen z. B. mit fachärztlichen Selek- 
tivverträgen nach $\$ 73 \mathrm{c}$ und weiteren Versorgungsformen, zu einer stärker die Sektoren übergreifenden Gesamtversorgung beitragen. Ziel war es, einen umfassenden, populationsorientierten Ansatz im Gegensatz zum traditionellen Anbieterund Sektor-bezogenen Gesundheitswesen zu stärken. Allerdings gab es in den letzten Jahren auch viele Herausforderungen zu meistern und Rückschläge zu verkraften: Restriktivere Vorgaben der Aufsichtsbehörden einerseits behinderten die weitere Umsetzung innovativer Versorgungsprojekte, die Krankenkassen agierten darauf hin zurückhaltender bei der Finanzierung neuer Modelle.

Auch sog. "telemedizinische Versorgungskonzepte" wurden durch gesetzliche Krankenkassen häufig über IV-Verträge ermöglicht, z. B. mit der Zielgruppe der Patienten mit einer Herzinsuffizienz. Telemedizin umfasst im Allgemeinen diagnostische und therapeutische Methoden unterschiedlicher medizinischer Fachrichtungen, die mittels Telekommunikation die räumliche oder zeitliche Distanz zwischen Arzt und Patient oder zwischen sich konsultierenden Ärzten überbrücken helfen. Klassische telemedizinische Anwendungen sind z. B. das Monitoring klinischer Parameter oder der Medikationsadhärenz bei chronisch kranken Patienten, die Unterstützung der Akutversorgung durch regionale Schlaganfallnetzwerke sowie die Vermeidung von Behandlungsbrüchen bei Sektoren-Übergängen, z. B. nach Krankenhausentlassung. Die Entwicklung telemedizinischer oder e-Health-gestützter Versorgungskonzepte für neue Patientengruppen und Indikationen findet hingegen nur im bescheidenen Umfang statt, obwohl es in der regionalen Versorgung in ländlichen Räumen einen hohen Bedarf an innovativen Lösungen gibt. Auch ist die Überführung in die Regelversorgung häufig schwierig.

Gesetzlich Krankenversicherte mit einer unheilbaren und lebensverkürzenden Erkrankung haben schließlich seit 2007 einen rechtlich verankerten Anspruch auf zusätzliche Unterstützung im Sinne einer „Spezialisierten Ambulanten Palliativversorgung" (SAPV). SAPV muss ärztlich verordnet und muss durch die Komplexität der Behandlungssituation begründet sein. Hier finden sich im bundesweiten
Vergleich teils erhebliche Unterschiede in der Vergütung, die bislang die Vergleichbarkeit erschweren und zu Unterschieden in der Versorgungsgestaltung führen.

Mit dem Gesetz zur Verbesserung der Versorgungsstrukturen in der gesetzlichen Krankenversicherung (GKV-VStG) wurden 2012 umfassende Maßnahmen auf den Weg gebracht, die eine gute und flächendeckende Versorgung auch für die Zukunft sichern sollten. Hier wurde z. B. die Möglichkeit einer Anerkennung sowie einer Förderung von Arztnetzen geschaffen. Arztnetze sind regionale Zusammenschlüsse von Haus- und Fachärzten, die mit weiteren Anbietern aus dem Gesundheitswesen, z. B. Pflege oder einer Klinik, strukturiert zusammenarbeiten. Interessant ist, dass offenbar etwa ein Drittel aller niedergelassenen Ärzte aktuell in einem der 400 deutschen Arzt- bzw. Praxisnetze mitarbeitet. Allerdings ist davon auszugehen, dass sich Arztnetze hinsichtlich ihrer Ausrichtung und ihres Entwicklungsstandes stark unterscheiden. Insofern ist bedeutsam, dass im GKV-VStGesetz verbindliche Versorgungsziele und Qualitätsanforderungen formuliert wurden, die professionelle Praxisnetze erfüllen müssen. Innovative Netzinitiativen wie das bekannte "Gesunde Kinzigtal“ verbinden Voraussetzungen eines professionellen Praxisnetzes mit den Vertragsmodalitäten einer Integrierten Versorgung und übernehmen die Organisationsverantwortung für eine populationsbasierte regionale Versorgung.

\section{Die Beiträge zum Schwerpunkt}

Das Schwerpunktheft hat zum Ziel, sowohl ausgewählte innovative Versorgungsmodelle als auch Modelle vernetzter Versorgung zu thematisieren: Der allgemeine Teil umfasst fünf Übersichten zum heutigen Stand der Disease-Management Programme, zur Integrierten und Hausarzt-zentrierten Versorgung, zu Ärztenetzen und zu telemedizinischen Ansätzen in Deutschland. Im spezifischen Teil werden in Anwendungsfeldern sechs Projekte mit einbezogenen Partnern und unterschiedlichen Indikationsbereichen vertiefend beschrieben. Schwerpunkte liegen auf ausgewählten Modellen der Integrierten Versorgung und ihrer Evaluation, ei- nerseits eines populationsbezogenen, andererseits eines indikationsbezogenen IVModells. Vertiefend werden drei Evaluationsansätze zur Qualität der Hausarzt-zentrierten Versorgung in Baden-Württemberg, der IV bei schweren psychischen Erkrankungen und einem Stepped-CareAnsatz zur Implementierung der Nationalen Versorgungs-Leitlinie Unipolare Depression beschrieben. Abschließend wird die Vernetzung von Sektoren, Disziplinen und Professionen am Beispiel der spezialisierten ambulanten Palliativversorgung (SAPV) dargestellt.

$R$. Linder und Kollegen vom Wissenschaftliches Institut der Techniker Krankenkasse geben in ihrem Beitrag zunächst einen Überblick zur Einführung der Disease Management Programme (DMP) und der tatsächlichen Inanspruchnahme sowie den Programmkosten. Primär fokussieren sie Schwierigkeiten bei der Nutzenbewertung der in Deutschland verfügbaren DMP. Da bislang keine prospektive randomisiert-kontrollierte Studie zu DMPs besteht, werden Ergebnisse dreier Studien zusammengefasst, welche Routinedaten der gesetzlichen Krankenversicherung nutzten.

Volker Amelung und Kollegen des Bundesverbands Managed Care e. V. analysieren in ihrem Artikel, auf der Grundlage leitfadengestützter Interviews mit Vorständen und Verantwortlichen für die Integrierte Versorgung, wie Krankenkassen die derzeitigen Regelungen und Realisierungen der Integrierten Versorgung wahrnehmen und welche fördernden bzw. hindernden Faktoren bestehen. Aufgrund der Erfahrungen der letzten Jahre schlussfolgern die Autoren, dass die Rahmenbedingungen für IV dringend weiterentwickelt und innovationsfreundlicher gestaltet werden sollten.

Im Beitrag von Robert Lübeck, Martin Beyer und Ferdinand Gerlach vom Institut für Allgemeinmedizin am Universitätsklinikum Frankfurt wird die „Hausarzt-zentrierte Versorgung“ (HzV) fokussiert. In der Arbeit werden auf der Basis einer Literaturrecherche und Anfragen bei Vertragsteilnehmern einerseits Prinzipien und Rahmenbedingungen der $\mathrm{HzV}$ erläutert und andererseits die Verbreitung dieser Versorgungsform beschrieben. Die Autoren sind überzeugt, dass die Schaf- 
fung eines leistungsfähigen primärärztlichen Systems durch die $\mathrm{HzV}$ begünstigt und entschieden vorangetrieben werden kann.

Neeltje van den Berg und Co-Autoren von der Universität Greifswald beschäftigen sich mit Versorgungskonzepten mit telemedizinischen Funktionalitäten, denen für ländliche Regionen mit einer geringen Bevölkerungsdichte und drohenden Lücken in der wohnortnahen Versorgung eine besondere Bedeutung zur Unterstützung der medizinischen Versorgung zukommt. Obwohl zahlreiche Szenarien für telemedizinische Ansätze erprobt werden, werden von den Autoren die weiterhin bestehende unzureichende Entwicklung geeigneter Evaluationsdesigns zur Wirksamkeit und der gesundheitsökonomischen Effekte telemedizinischer Konzepte bemängelt.

Zwei in der Versorgung aktive Hausärzte, Veit Wambach und Jörg Lindenthal, zeigen in ihrem Beitrag am Beispiel eines Gesundheitsnetzes in Nürnberg auf, wie es Arztnetzen gelingen kann, ein Plus an Effizienz bei gleichzeitig überdurchschnittlicher Versorgungsqualität und hoher Patientenzufriedenheit zu erreichen. Die Berücksichtigung regionaler Besonderheiten, die Nähe zum Patienten und eine konsequent gelebte Patientenorientierung stellen dabei aus Sicht der Autoren und Daten aus dem aktiven Netz die entscheidenden Erfolgsfaktoren für erfolgreiche Arztnetze dar.

Das Modell der Integrierten Versorgung „Gesundes Kinzigtal“ (IVGK) gehört bundesweit zu den bekanntesten innovativen Versorgungsmodellen in Deutschland und wird mithilfe verschiedener quantitativer und qualitativer Methoden wissenschaftlich evaluiert. Helmut Hildebrandt und Co-Autoren beschreiben in ihrem Beitrag sowohl Ergebnisse zur Verbesserung des Gesundheitsstatus, zum Erleben einer besseren Versorgung durch die Versicherten als auch zum optimierten Ressourceneinsatz in der Region. Dabei weist die Mehrzahl der evaluierten Indikatoren zur Versorgungsqualität auf eine positive Entwicklung, bei den Kosten zeigt sich über alle Jahre ein positives Ergebnis gegenüber der Vergleichspopulation.
Im Beitrag von Claus-Werner Wallesch von der neurologischen Rehabilitationsklinik in Elzach werden die Versorgung, Versorgungsdefizite, Unterstützungsbedarfe und deren Deckungsmöglichkeiten in der Versorgung von Schlaganfallpatienten in der akuten und chronischen Phase analysiert. Nach Einschätzung des Autors bestehen Versorgungsdefizite vor allem in der wohnortnahen, multidisziplinären, teilhabeorientierten Versorgung und der fehlende Einbindung rehabilitationserfahrener Fachärzte. Die Defizite sind vor allem durch fehlende Finanzierungsmöglichkeiten durch die gesetzlichen und privaten Krankenkassen im ambulanten Sektor begründet.

Gunter Laux und Kollegen der Institute für Allgemeinmedizin an den Universitätskliniken Heidelberg und Frankfurt fokussieren auf die Beantwortung der Fragen, wie sich die Versorgung in der Hausarztzentrierten Versorgung auf darin eingeschriebene Versicherte am Beispiel des Modells der AOK Baden-Württemberg und deren Versorger auswirkt. Die methodische Vorgehensweise der Evaluation folgt einem Mixed-Methods-Design, bei dem neben quantitativen auch qualitative Verfahren zum Einsatz kamen. Größtenteils lassen sich positive Assoziationen zwischen einer Einschreibung in die $\mathrm{HzV}$ und den jeweils untersuchten Zielgrößen beobachten, die dafür sprechen, die $\mathrm{HzV}$ sowie die dazu korrespondierende Begleitevaluation weiterzuführen.

Im folgenden Beitrag von Martin Lambert und Kollegen von der Klinik für Psychiatrie und Psychotherapie am Universitätsklinikum Hamburg-Eppendorf wird das sog. „Hamburger Modell“, ein Integriertes Versorgungsmodell nach $\$ 140$ SGB V für Psychose-Betroffene, beschrieben. Im Rahmen einer Qualitätssicherungsstudie wurden patientenbezogene Daten zum Aufnahmestatus, zum Verlauf der Erkrankung und der in Anspruch genommenen Leistungen erfasst. Es wird gezeigt, dass durch eine spezifische Integrierte Versorgung auch bei schwer erkrankten Psychose-Patienten eine multidimensionale Verbesserung und Stabilisierung der Erkrankung erreicht werden kann.

Leitlinien empfehlen bei der Depressionsversorgung abgestufte und interdisziplinäre Versorgungsmodelle. International erprobte Collaborative und Stepped Care gelten als evidenzbasierte Modelle für die Versorgung von depressiven Patienten. Martin Härter und Kollegen des Instituts und der Poliklinik für Medizinische Psychologie vom Universitätsklinikum HamburgEppendorf beschreiben in ihrer Arbeit zunächst allgemein Wirksamkeit und Effizienz dieser Ansätze. Am Beispiel des Gesundheitsnetzes Depression im Hamburger Netzes psychische Gesundheit (www. psychenet.de) wird beschrieben, wie eine leitliniengerechte gestufte Depressionsdiagnostik und -behandlung unter Einbezug innovativer niedrigschwelliger Interventionen gemeinsam mit Hausärzten, Psychotherapeuten und Psychiatern sowie Kliniken umgesetzt werden kann und welche Vorteile für die teilnehmenden Partner und Patienten zu erwarten sind.

Bernd Alt-Epping und Friedemann Nauck aus der Klinik für Palliativmedizin der Universität Göttingen beschäftigen sich in ihrem Beitrag mit der „Spezialisierten Ambulanten Palliativversorgung “ (SAPV), welche als ein intensiviertes, multiprofessionelles palliativmedizinisches Unterstützungsangebot für schwer kranke und sterbende Patienten in besonders komplexen Belastungssituationen entwickelt wurde. Trotz der G-BA-Richtlinie lässt sich in Deutschland ein regional sehr divergierendes Bild über die Umsetzung der SAPV darstellen. Ein spezifischer Fokus wird von den Autoren auf die rechtlichen und konzeptuellen Rahmenbedingungen, finanzielle und klinische Aspekte sowie die Perspektiven für eine zukünftige, umfassende palliative Betreuung komplex belasteter Patienten gelegt.

\section{Ausblick}

Mit dem GKV-Versorgungsstärkungsgesetz unternimmt die Bundesregierung aktuell einen erneuten Anlauf zur Stärkung innovativer Versorgungsmodelle. Es ist erklärtes Ziel dieses Gesetzesvorhabens, durch spezifische Maßnahmen eine bedarfsgerechte, flächendeckende und gut erreichbare medizinische Versorgung auf hohem Niveau erreichen zu wollen (s. Kabinettsentwurf zum Versorgungsstärkungsgesetz, [1]). Hierzu gehören insbesondere die Flexibilisierung und Verbesserung der Rahmenbedingungen für die Tätigkeit in 
der vertragsärztlichen Versorgung, z. B. durch die Förderung der Versorgungsorientierung der Vergütungsregelungen sowie die angemessene Vergütung der Leistungen der Hochschulambulanzen. Auch sollen Wartezeiten auf Facharzttermine verringert und die PsychotherapieRichtlinien des Gemeinsamen Bundesausschusses überarbeitet werden. Darüber hinaus soll der Gestaltungsspielraum der Krankenkassen insbesondere beim Abschluss von Verträgen im Wettbewerb vergrößert werden.

Das wirklich Interessante für die weitere Entwicklung neuer Versorgungsformen ist der Plan, dass ab 2016 Innovationen in der Versorgung mit jährlich 225 Mio. $€$ und die sie begleitende Versorgungsforschung mit 75 Mio. $€$ pro Jahr durch die Schaffung eines dafür vorgesehenen Fonds gefördert werden sollen. Dieser Innovationsfond hat aufgrund der Quantität und Qualität der Maßnahmen ein großes Potential. Es könnten erstmals, mit angemessenen Mitteln über längere Zeit, komplexe Interventionen in der Versorgung entwickelt, pilotiert, verbessert, evaluiert und umgesetzt werden, wie es schon seit vielen Jahren von der Wissenschaft national wie international gefordert wird. Aktuell fehlen z. B. gelingende Ansätze und Studien zur Implementierung und Evaluation von evidenzbasierten Leitlinien oder zur systematischen Überprüfung innovativer Versorgungsformen, das zeigen eindrucksvoll die Beiträge in diesem Schwerpunktheft.

Es besteht eine dringende Notwendigkeit adäquater Versorgungs- und Forschungsstrukturen bzw. -bedingungen, die eine Implementierung solch komplexer Interventionen ermöglichen und die gleichzeitig den Verwertungszusammenhang im Auge behalten ([2, 3]). Hier sind verbindliche Design- und Evaluationskriterien notwendig, die bei positiven Ergebnissen innovativer Versorgungsformen die Basis für eine Translation in die Routineversorgung bilden können. Dazu ist es sicher auch angebracht, Evaluationen von Versorgungsinnovationen in einem internationalen Kontext zu betrachten, denn die Versorgungsfragen, die sich im deutschen Gesundheitswesen stellen, stellen sich anderorts in gleicher oder sehr ähnlicher Weise. Hierdurch kann die deutsche
Wissenschaftslandschaft entscheidend weiterentwickelt und im internationalen Vergleich auf eine Stufe mit anderen Ländern (z. B. USA, Großbritannien) gestellt werden, in denen Outcome Research bzw. Health Services Research schon seit mehreren Jahren zum Instrumentarium der Klinischen Wissenschaft und Gesundheitsforschung gehört (s. Stellungnahme des Deutschen Netzwerk Versorgungsforschung [4]). Das deutsche Versorgungssystem kann mit einem intelligenten Einsatz dieser Mittel zeigen, dass es gewillt ist, dies zu fördern und sich als stärker lernfähig als bisher zu beweisen. Ziel sollte sein, dass qualitativ hochwertige, innovative und evidenzbasierte Versorgungsansätze, die einer unabhängigen qualitativen Begutachtung standhalten, unseren Bürgerinnen und Bürgern zugutekommen. Wenn es dann in der Folge gelingt, erfolgreich umgesetzte und evaluierte Ansätze viel stärker als bisher in der Routineversorgung zu etablieren, dann kommen wir einem evidenzbasierten und patientenzentrierten Gesundheitswesen wesentlich näher.

Die in diesem Schwerpunktheft enthaltenen Beiträge zeigen den Stand der erreichten Fortschritte und offenen Fragen bei innovativen und vernetzten Versorgungsmodellen beispielhaft auf. Ihre Diskussion kann die anstehenden Aufgaben und Entwicklungen und die dazu notwendige Versorgungsforschung inhaltlich unterstützen. In diesem Sinne bedanken wir uns bei allen Autorinnen und Autoren für die interessanten Beiträge und die zeitnahe Finalisierung. Und nun wünschen wir Ihnen, den Leserinnen und Lesern eine spannende Lektüre,

Ihre
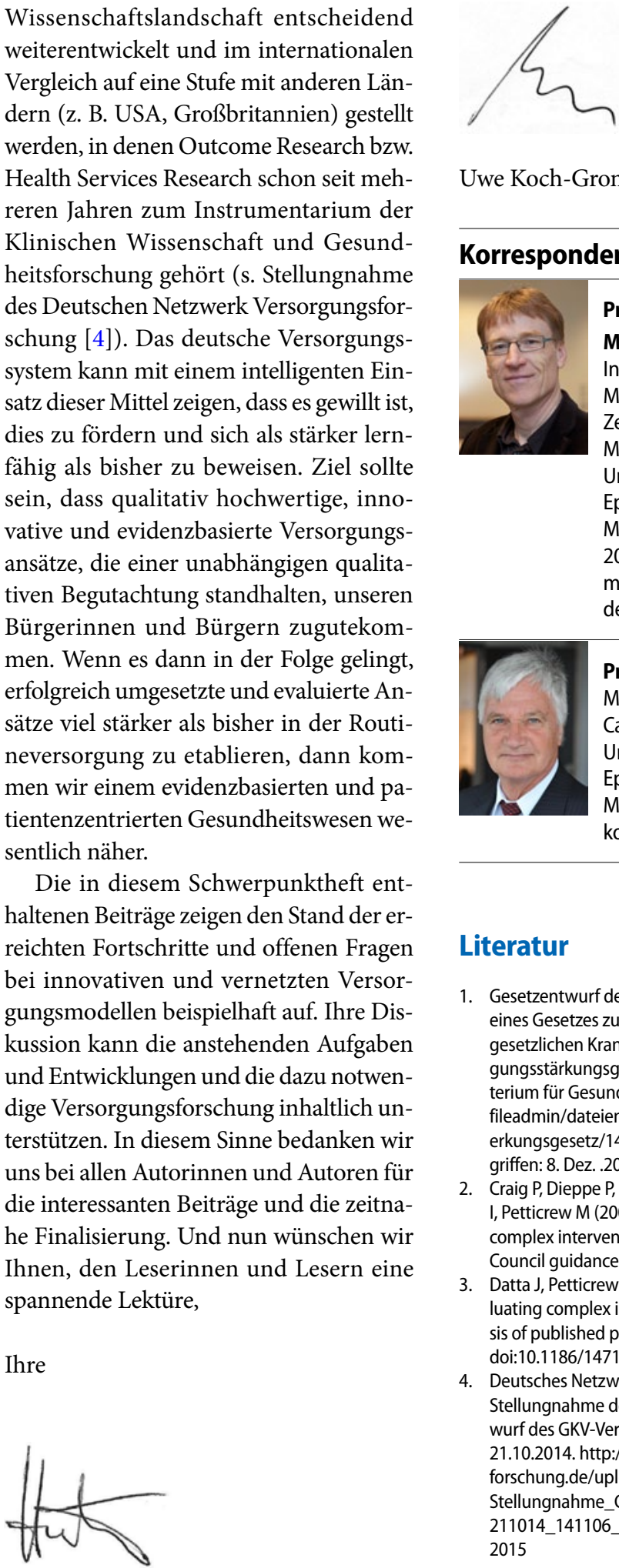

Uwe Koch-Gromus

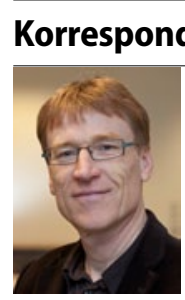

Prof. Dr. med. Dr. phil.

M. Härter Dipl. Psych.

Institut und Poliklinik für

Medizinische Psychologie

Zentrum für Psychosoziale

Medizin

Universitätsklinikum Hamburg-

Eppendorf

Martinistr. 52 (W26)

20246 Hamburg

m.haerter@uke.uni-hamburg. de

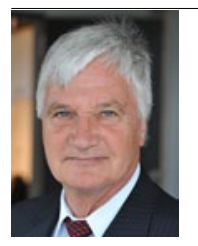

Prof. Dr. Dr. U. Koch-Gromus Medizinische Fakultät Campus Lehre, N 55 Universitätsklinikum HamburgEppendorf

Martinistr. 52, 20246 Hamburg koch@uke.uni-hamburg.de

\section{Literatur}

1. Gesetzentwurf der Bundesregierung Entwurf eines Gesetzes zur Stärkung der Versorgung in der gesetzlichen Krankenversicherung (GKV-Versorgungsstärkungsgesetz - GKV-VSG). Bundesministerium für Gesundheit. http://www.bmg.bund.de/ fileadmin/dateien/Downloads/N/Versorgungsstaerkungsgesetz/141217_Entwurf_VSG.pdf. Zugegriffen: 8. Dez. 2014

2. Craig P, Dieppe P, Macintyre S, Michie S, Nazareth I, Petticrew M (2008) Developing and evaluating complex interventions: the new Medical Research Council guidance. BMJ 337:a1655

3. Datta J, Petticrew M (2013) Challenges to evaluating complex interventions: a content analysis of published papers. BMC Public Health 13:568. doi:10.1186/1471-2458-13-568

4. Deutsches Netzwerk Versorgungsforschung e. V. Stellungnahme des DNVF zum Referentenentwurf des GKV-Versorgungsstärkungsgesetz vom 21.10.2014. http://www.netzwerk-versorgungsforschung.de/uploads/Stellungnahmen/DNVFStellungnahme_GKV-VSG-Referentenentwurf211014_141106_R-Korr.pdf. Zugegriffen: 2. März 2015

Martin Härter und 